\title{
Initial stage of the active mode-locking in semiconductor heterolasers
}

\author{
Alexandre S. Shcherbakov ${ }^{1)}$, Alexey Yu. Kosarsky ${ }^{2)}$, Joaquin Campos Acosta ${ }^{3)}$, \\ Pedro Moreno Zarate ${ }^{1)}$, and Svetlana Mansurova ${ }^{1)}$ \\ 1) National Institute for Astrophysics, Optics and Electronics, Puebla, 72000, Mexico. \\ Phones: 52 (222) 266 3100, ext. 2205, 9308 \& 2206; \\ E-mail: alex@inaoep.mx,pemzamx@gmail.com, smansur@inaoep.mx \\ 2) LLC Petro, Peterhoff Chausse 71, Saint-Petersburg, 198206, Russian Federation. \\ Phone: 7 (812) 534 3334; E-mail: alexey.kosarsky@jt-int.com \\ 3) CSIC-Institute for Applied Physics, Serrano 144, Madrid, 28006, Spain. \\ Phone: 34 (91) 561 8806, ext. 206; E-mail: joaquin.campos@ifa.cetef.csic.es
}

\begin{abstract}
We make an attempt to develop a novel approach to describing the initial stage of the active mode-locking in semiconductor laser structures based on analyzing the properties of dispersion relations in terms of stability for small initial perturbations. Nonlinear process of shaping optical pulses is interpreted as manifesting instability of diffusion type. For the purposes of experimental investigations, the auto-manual opto-electronic measuring system detecting average time parameters inherent in ultra-short optical pulse trains has been designed. This system is able to register auto-correlation functions of the second order exploiting the interferometric technique as well as to identify a pulsed character of the incoming light radiation. Experimental confirmations of appearing the diffusive instability within the active mode-locking process in semiconductor laser structures operating in the near infrared range are presented.
\end{abstract}

Key words: Active mode-loking, semiconductor heterolaser, diffusion instability, detection system, train-average time parameters

\section{INTRODUCTION}

A novel approach to describing the initial stage of the active mode-locking in semiconductor heterolasers is proposed. It is based on analyzing properties of dispersion relations in terms of stability for small initial perturbations. Due to the light field in active medium is governed by the nonlinear Ginzburg-Landau diffusive equation, the dispersion relation between the wave number $\mathbf{k}$ and the frequency $\omega$ involves the light amplitude and has the simple root, including additional controlling parameter $\boldsymbol{\mu}$. When $\operatorname{Im} \mathbf{k}(\boldsymbol{\omega}, \boldsymbol{\mu}) \neq \mathbf{0}$, one can observe the diffusive instability accompanied by the growth ( $\operatorname{Im} \mathbf{k}<\mathbf{0})$ or attenuation ( $\operatorname{Im} \mathbf{k}>\mathbf{0})$ of light field; with $\operatorname{Im} \mathbf{k}=\mathbf{0}$, the neutral stability appears. Because Im $\mathbf{k}$ exceeds zero with one set of $\mu$ or it is under zero for the other set of $\mu$, one can consider the dependence $\mu=\mathbf{G}(\omega)$ that gives a boundary curve dividing the areas of stability and instability in the $(\omega ; \mathbf{k})$-space. If each optical pulse, incoming into the active medium, is coinciding with maximum gain, one can find $\mu=-\alpha_{0}+\alpha_{1}-\alpha_{2}$, where $\alpha_{0}, \alpha_{1}$, and $\alpha_{2}$ are the factors of attenuation, gain, and absorption. Then, $\mu$ has meaning of the pure gain at a center of optical pulse and gives a parabolic boundary curve in the $(\omega ; \mathbf{k})$-space. The point $\left(\mu_{\mathbf{C}}, \omega_{\mathbf{C}}\right)$ of a minimum on that boundary curve is a critical point. Exceeding the critical value $\mu_{\mathbf{C}}=\mathbf{0}$, the parameter $\boldsymbol{\mu}$ determines the band of unstable frequencies near a critical frequency $\omega_{\mathbf{C}}=\omega_{\mathbf{0}}$. Originating unstable frequencies, extracting energy from the medium, governs growing the amplitude of initial perturbation. Therefore, a condition of smallness for the amplitude no longer obeys, that nonlinearity comes into force and competes with dispersion effects, restricting the amplitude and shaping the optical pulse with rather stable envelope. The frequency $\omega_{\mathbf{C}}$ is preferable and plays a role

Photonics North 2009, edited by Réal Vallée, Proc. of SPIE Vol. 7386, $73862 Z$ (C) 2009 SPIE · CCC code: 0277-786X/09/\$18 - doi: 10.1117/12.839296 
of the resonant frequency, because just it is the first, which turns to be unstable. Thus, nonlinear process of shaping optical pulses is interpreted as manifesting instability of diffusion type. Experimental confirmations of appearing the diffusive instability within the active mode-locking process in InGaAsP/InP structures are presented. There are two thresholds in semiconductor heterolasers operating in the active mode-locking regime, namely, one can recognize the threshold of spike-mode oscillation and the threshold of pulse shaping.

\section{GENERAL THEORETICAL CONSIDERATION}

Together with the spectral description ${ }^{\mathbf{1}}$ for a process of the active mode-locking in semiconductor laser structures, recently rather adequate models, based on considering the pulse evolution during sequential passing through the domains with optical gain and absorption ${ }^{2-4}$, have been proposed. This paper is devoted to describing the initial stage of the above-mentioned processes via studying the properties of dispersion relations in terms of stability for small initial perturbations. For a slowly varying wave packet

$$
A(z, t)=A_{0}(z, t) \exp [i \theta(z, t)]
$$

one can introduce the local angular frequency $\omega(\mathbf{z}, \mathbf{t})=-\partial \theta / \partial \mathbf{t}$ and the local wave number $\mathbf{k}(\mathbf{z}, \mathbf{t})=\partial \theta / \partial \mathbf{z}^{5}$. Because the field $\mathbf{A}(\mathbf{z}, \mathbf{t})$ in a medium is governed by some evolution equation, the functional dependence between $\mathbf{k}$ and $\omega$ can be found; and this functional dependence represents the dispersion relation, which can be written generally as $\mathbf{P}(\omega ; \mathbf{k})=\mathbf{0}$. If a medium exhibits nonlinearity, the corresponding dispersion relation includes the dependence on the amplitude $\mathbf{A}_{\mathbf{0}}$ of wave packet

$$
\mathbf{P}\left(\omega ; \mathbf{k} ; \mathbf{A}_{\mathbf{0}}\right)=\mathbf{0}
$$

The physical system under consideration, i.e. semiconductor laser structure, includes the optical gain, so that Eq.(2) represents the complex-valued relation between $\mathbf{k}$ and $\boldsymbol{\omega}$, which has the simple complex-valued root

$$
\mathbf{k}(\omega, \mu)=\mathbf{k}_{\mathbf{R}}(\omega, \mu)+\mathbf{i} \mathbf{k}_{\mathbf{I}}(\omega, \mu),
$$

where $\mu$ is an additional controlling parameter ${ }^{\mathbf{6}}$. With exploiting Eq.(3), Eq.(1) for a wave packet takes the form.

$$
A(z, t)=A_{0}(z, t) \exp \left[i\left(k_{R} z-\omega t\right)\right] \exp \left(-k_{I} z\right)+c . c .
$$

Evolution equation, which governs the field $\mathbf{A}(\mathbf{z}, \mathbf{t})$ described by Eq.(4), has the character of diffusion equation. When $\mathbf{k}_{\mathbf{I}}(\omega, \mu) \neq \mathbf{0}$, one can observe the diffusive instability being accompanied by exponentional growth (with $\mathbf{k}_{\mathbf{I}}(\omega, \mu)<\mathbf{0}$ ) or attenuation (with $\mathbf{k}_{\mathbf{I}}(\omega, \mu)>\mathbf{0}$ ) of field amplitude. In the case of $\mathbf{k}_{\mathbf{I}}(\omega, \mu)=\mathbf{0}$, the neutral stability can be observed. Because $\mathbf{k}_{\mathbf{I}}(\omega, \mu)$ can exceed zero with one set of values of the parameter $\mu$ as well as it can be under zero for the other set of $\mu$, one can formally consider the frequency dependence $\mu=\mathbf{G}(\omega)$, which gives a boundary curve dividing areas of stability and instability in the $(\omega ; \mathbf{k})$-space. To analyze the behavior of systems under consideration from the viewpoint of their stability relative to small initial perturbations let us exploit an approximation of one-directional (along, for example, positive direction of the z-axis) traveling for the light wave. This process is described by the following inhomogeneous evolution equation

$$
\frac{\partial \mathbf{A}}{\partial \mathbf{z}}+\frac{1}{\mathbf{c}} \frac{\partial \mathbf{A}}{\partial \mathbf{t}}=\mathbf{f}(\mathbf{A}, \mathbf{z}, \mathbf{t})
$$


where $\mathbf{c}$ is the group velocity, while $\mathbf{f}(\mathbf{A}, \mathbf{z}, \mathbf{t})$ is some function reflecting possible contributions from the gain, dispersion, and nonlinearity. Now, one can provide the analysis of dispersion relations for the solutions to Eq.(5) in the form of

$$
A(z, t)=b \exp \left\{i\left[k-k\left(\omega_{0}\right)\right] z-\left[\omega-\omega_{0}\right] \mathbf{t}\right\}+\text { c.c. }
$$

where $\mathbf{b}$ and $\omega_{\mathbf{0}}$ is small amplitude and the current frequency of a wave packet. To illustrate the proposed approach let us consider an example of the Ginzburg-Landau diffusive evolution equation, which appears usually during the analysis of various mode-locking problems ${ }^{7}$. In similar particular cases, the right side of Eq.(5) takes the form

$$
\mathbf{f}(\mathbf{A}, \mathbf{z}, \mathbf{t})=-\alpha \mathbf{A}-\mathbf{i}\left(\beta \frac{\partial^{2} \mathbf{A}}{\partial \mathbf{t}^{2}}+\gamma|\mathbf{A}|^{2} \mathbf{A}\right)
$$

where $\alpha, \beta$, and $\gamma$ are the factors of the gain (losses), dispersion, and nonlinearity, respectively. Substitution of Eq.(6) into Eq.(5) with the right side of Eq.(7) leads to the complex-valued dispersion relation

$$
\mathbf{k}=\mathbf{k}\left(\omega_{0}\right)+\frac{\omega-\omega_{0}}{\mathbf{c}}+\beta\left(\omega-\omega_{0}\right)^{2}-\gamma|\mathbf{b}|^{2}+\mathbf{i} \alpha
$$

which represents by it self an explicit function $\mathbf{k}$ of $\omega$ being similar to Eq.(3). In Eq.(8), one can separate the controlling parameter $\mu=\alpha$ that determines the boundary curve, namely, the abscises axis in the $(\omega ; \mathbf{k})$-space. It is seen from Eq.(8) that the system becomes to be diffusively unstable with $\boldsymbol{\alpha} \neq \mathbf{0}$.

Now, let us turn our attention to the process of active mode-locking in semiconductor laser at the approximation that each ingoing the optical pulse into an active medium is coinciding with a maximum of gain. In this case ${ }^{2}$, the right hand side of Eq.(5) takes the form

$$
\mathbf{f}(\mathbf{A}, \mathrm{z}, \mathbf{t})=\left\{-\alpha_{0}+\alpha_{1}\left[1-\mathbf{M}\left(1-\cos \omega_{m} t\right)\right]-\alpha_{2}\left(1-\frac{1}{\omega_{S}^{2}} \frac{\partial^{2}}{\partial \mathbf{t}^{2}}\right)\right\} \mathbf{A},
$$

where $\mathbf{M}$ and $\omega_{\mathrm{m}}$ are the depth and frequency of modulation; $\alpha_{0}, \alpha_{1}$, and $\alpha_{2}$ are the factors of the attenuation, gain, and saturable absorption, respectively; $\omega_{\mathbf{S}}$ is the amplification (absorption) bandwidth. In so doing, smallness of the amplitude inherent in the initial perturbation is taken into account through exploiting the weak mode-locking approximation within deriving Eq.(9). Similar to the above-considered example, the dispersion relation, corresponding Eq.(5) with the right hand side in the form of Eq.(9), represents an explicit complex-valued function

$$
\mathbf{k}=\mathbf{k}\left(\omega_{0}\right)+\frac{\Delta \omega}{\mathbf{c}}-\mathbf{i}\left\{-\alpha_{0}+\alpha_{1}\left[1-M\left(1-\cos \omega_{m} \mathbf{t}\right)\right]-\alpha_{2}\left(1-\frac{\Delta \omega^{2}}{\omega_{S}^{2}}\right)\right\}
$$

where $\Delta \omega=\omega-\omega_{0}$. It follows straightly from Eq.(10) that within the regime of active mode-locking the combined system "field-medium" allows appearing the diffusive instability with the controlling parameter

$$
\mu=-\alpha_{0}+\alpha_{1}-\alpha_{2}
$$

Thus, one can separate the controlling parameter $\mu$, which has a meaning of the pure gain at a center of the incoming optical pulse and determine a parabolic-like boundary curve in the $(\omega ; \mathbf{k})$-space, see Fig.1a. The point of a minimum 
$\left(\mu_{\mathbf{C}}, \omega_{\mathbf{C}}\right)$ on that boundary curve is a critical point. Exceeding the critical value $\mu_{\mathbf{C}}=\mathbf{0}$ by the controlling parameter determines the band of unstable frequencies in the vicinity of a critical frequency $\omega_{\mathbf{C}}=\omega_{\mathbf{0}}$. Originating the band of unstable frequency components, which are able to extract energy from active medium, governs growing the amplitude of initial perturbation. By this is meant that the condition of smallness for the amplitude no longer obeys, and the nonlinearity comes into force and competes with dispersion effects, resulting in the restriction of growing the amplitude and in shaping the optical pulse with rather stable envelope. The frequency $\omega_{\mathbf{C}}$ (or the mode $\omega_{\mathbf{C}}$, if periodic boundary conditions are introduced) is preferable, because just this frequency component is the first, which turns to be unstable. Physically, just this frequency plays a role of the resonant frequency (or mode) in the system under consideration. Figure $1 \mathrm{~b}$ presents the results of computer simulation illustrating the development of initially lowpower seeding optical fluctuation in semiconductor laser structure with the diffusive instability.

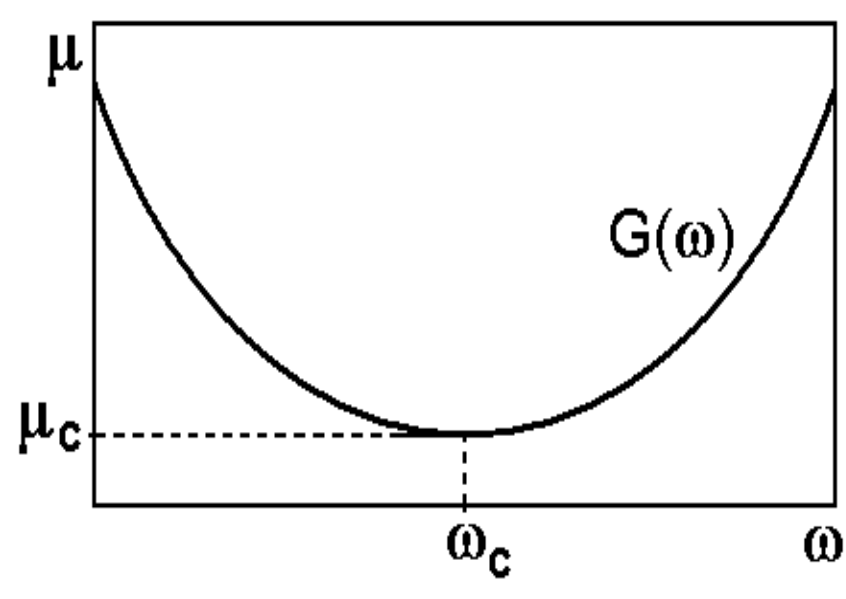

a.

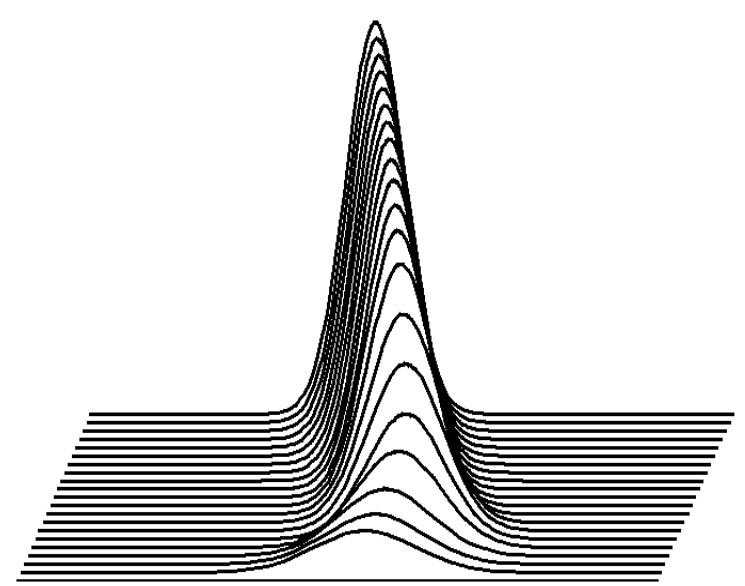

b.

Figure 1. Exhibiting the diffusive instability. (a) The boundary curve $\mathbf{G}(\omega)$; an area lying above $\mathbf{G}(\omega)$ corresponds to growing the initial perturbation, while an area placing below is associated with attenuating the initial perturbations;

(b) The evolution of an initial perturbation, i.e. the dynamics of pulse growing and stabilizing.

The parameters inherent in active medium had been chosen in such a way that the magnitude of the controlling parameter exceeded a critical value. One can see a stage of growing the seeding fluctuation amplitude at the expense of the energy from active medium as well as a stage of stabilizing the optical pulse envelope due to a balance between contributions of the dispersion and the nonlinearity.

\section{DETECTION SYSTEM OF AVERASGE TIME PARAMETERS FOR CONTINUOUS TRAINS OF ULTRA-SHORT OPTICAL PULSES IN NEAR-INFRARED RANGE}

To study continuous trains of ultra-short optical pulses the auto-correlation approach in parallel light beams was used. For this purpose an auto-manual opto-electronic system for detecting train-average time parameters had been created. The scheme of this detection system consists of optical auto-correlator 1, electronic controller 2, and a pair of the checking units 3, see Fig.2. The computer soft admits several regimes for the scheme operation: a) calibration of optical auto-correlator, b) the measuring cycle, and c) data processing, i.e. pulse width calculation, and data display. A high-repetition-rate ultra-short pulse trains arrive at the optical auto-correlator, i.e. at a two-beam scanning Michelson interferometer, which is formed by two total internal reflection prisms and a 50\%-mirror. The selection of prisms as the reflecting components leads to an opportunities of both to control the time distribution of light radiation simultaneously and to analyze the auto-correlation functions of the second order inherent in optical pulse trains. Moreover, this prismatic optical circuit permits keeping out the backward scattering from basic reflecting planes of the optical auto-correlator. The rays trace difference in the scanning interferometer is determined by relative disposition of the stationary prism as well as the moving prism, which is fixed on a long-path speaker. During the measuring cycle, 
the moving prism changes its position step-by-step in relation to one another. The scheme utilizes an interferometric technique of detection: the reflected optical signals form an interferogram, which is registered by a slow-speed photodetector. The obtenied photodetector output signal is amplified, then is converted into digital code, and finally goes into computer that controls the whole process. As a result, all the data related to cycle time-average autocorrelation function of pulse trains is stored in the computer memory and the train-average pulse width is calculated.



Figure 2. Scheme of the auto-manual opto-electronic detecting system for detecting average time parameters inherent in continuous high repetition rate trains of ultra-short optical pulses in near-infrared range

For an additional visual displaying of auto-correlation function the detected electronic signal arrives at an external memory oscilloscope. The checking units receive another part of pulsed radiation after interferometer. By using a highspeed photodetector and a sampling oscilloscope, one can observe the character of light radiation. The temporal picture is characterized by the fact that optical pulse interval is true, but the pulse envelope is determined by the response time inherent in a high-speed photodetector, whose bandwidth was about $3.5 \mathrm{GHz}$, so that adequate pulse width measuring was possible only when auto-correlation responses were exploited. This auto-manual opto-electronic scheme is acceptable for ultra-short pulse width measurements within the range from one to a few tens picoseconds, and the record time for an individual interferogram is no more than 1 second.

\section{THE ALGORITHM OF OPERATION}

The correlation methods of signal processing are successfully in use for a long time. In the simplest case of the second order correlations similar technique is based on the following rather general algorithm see Fig.3. The initial signal $\mathbf{S}(\mathbf{t})$ is applied to the input port 1 of a multiplier. The input port 2 is activated by either the additional signal $\mathbf{H}(\mathbf{t})$ or the same initial signal $\mathbf{S}(\mathbf{t})$, but they both have some variable temporal shift $\tau$ due to passing through a delay line and take the forms of $\mathbf{H}(\mathbf{t}+\tau)$ and $\mathbf{S}(\mathbf{t}+\tau)$, respectively. The product of a pair of the input signals is integrated with respect to time by the integrator. Thus, the auto- and cross-correlation functions are given by 

a) $G(\tau)=\int_{-\infty}^{\infty} \operatorname{dt} \mathbf{S}(\mathbf{t}) \mathbf{S}(\mathbf{t}+\tau)$
b) $K(\tau)=\int_{-\infty}^{\infty} \operatorname{dt} S(t) H(t+\tau)$.

These formulas represent the correlation functions of just the above-mentioned lowest possible order, i.e. the second one. Under some additional conditions, but definitely not always, the availability, for example, of the function $\mathbf{G}(\tau)$ makes it possible to identify the time dependence of the input signal $\mathbf{S}(\mathbf{t})$. If $\mathbf{H}(\mathbf{t})$ is extremely short pulse, which can be approximated by the Dirac $\delta$-function, and an area of this function $\mathbf{H}(\mathbf{t})$ is normalized to unity, one can find that $\mathbf{K}(\mathbf{t})=\mathbf{S}(\mathbf{t})$.

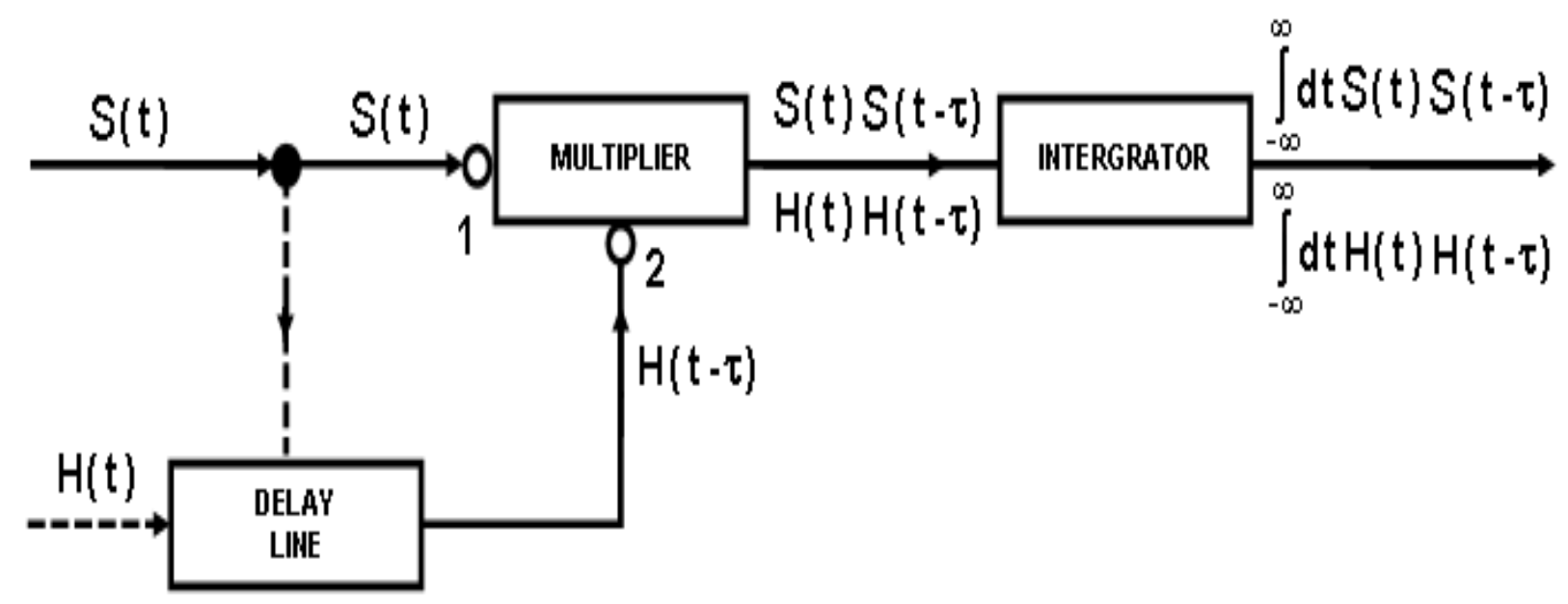

Figure 3. Principle scheme for shaping both auto- and cross-correlation functions.

The simplest optical auto-correlator, which can be exploited for estimating time parameters of ultra-short optical pulses in high-repetition-frequency trains, is a two-beam scanning Michelson interferometer with a slow-speed photodetector, see Fig.2 (part 1). In principle, it makes possible detecting the auto-correlation function for the light field strength and, after conversion, the Fourier spectral density of light radiation and find the train-average spectral width of radiation. Two fields $\mathbf{E}_{\mathbf{1}}(\mathbf{t})=\mathbf{E}(\mathbf{t})$ and $\mathbf{E}_{\mathbf{2}}(\mathbf{t})=\mathbf{E}(\mathbf{t}+\tau)$ related to ultra-short optical signals reflected from the stationary and moving mirrors, respectively, are summarized by a slow-speed photodetector. The delay time $\tau$ from pulse to pulse is varied by scanning the moving mirror under electronic control. The output signal is proportional to the energy $\mathbf{W}$ on a slow-speed photodetector under condition that the time of integration is long enough. This energy can be estimated as

$$
\text { a) } W \propto \int_{-\infty}^{\infty} \operatorname{dt}\left(E_{1}+E_{2}\right)^{2} \approx\left[G_{E}(0)+G_{E}(\tau)\right] \text {, b) } G_{E}(\tau)=\int_{-\infty}^{\infty} \operatorname{dt} E(t) E(t+\tau)=\frac{1}{2 \pi} \int_{-\infty}^{\infty} d \omega|E(\omega)|^{2} e^{-i \omega \tau} \text {, }
$$

where $\mathbf{G}_{\mathbf{E}}(\tau)$ is the auto-correlation function of the field strength, while $|\mathbf{E}(\omega)|^{2}$ is proportional to the Fourier spectral density of light radiation. However, during such a measurement (as well as with exploiting another Fourier spectrometers) the information about the phase of the field $\mathbf{E}(\omega)$ becomes to be lost. That is why one cannot make correct (unambiguous) conclusion about the train-average pulse width. The ultra-short optical pulse width can be determined from the spectral width rather accurately only if it is known in advance that optical pulse is spectrally (transform) limited, i.e. it does not include any internal frequency modulation. This takes place when the phase of field strength along the pulse width grows linearly, so that only the shape of optical pulse envelope determines the spectrum width. The half-width $\Delta \omega$ of the spectrum of power density for spectrally limited pulses and the half-width $\tau_{\mathbf{L}}$ for 
the dependence of power on time inherent in the spectrally (transform) limited pulses are connected by $\Delta \omega \cdot \tau_{L} /(2 \pi)=C_{B}$, where $C_{B}$ is the constant determined by the pulse shape. In general case, when pulse is not transform (spectrally) limited, the left hand side of the last formula exceeds $\mathbf{C}_{\mathbf{B}}$. To determine the train-average ultrashort optical pulse width, one needs two independent measures of the spectrum width and the internal frequency modulation. Within the direct photodetection, the time resolution is restricted by inertia of various components and an effect of storage associated with this inertia. The response function $\mathbf{R}(\mathbf{t})$, inherent in even rather high-speed photodetector, is not perfectly identical to the incoming optical signal $\mathbf{S}(\mathbf{t})$, because this response is conditioned by a transfer function $\mathbf{B}(\mathbf{t})$. As a result, one has to write

$$
R(t)=\int_{-\infty}^{\infty} d t_{1} S\left(t_{1}\right) B\left(t_{1}-t\right)
$$

in linear systems. Moreover, $\mathbf{B}\left(\mathbf{t}_{\mathbf{1}}-\mathbf{t}\right)=\mathbf{0}$ with $\mathbf{t}_{\mathbf{1}}>\mathbf{t}$ due to the causality principle. One can see that the response function $\mathbf{R}(\mathbf{t})$ is coinciding with the signal $\mathbf{S}(\mathbf{t})$ again only if the transfer function $\mathbf{B}(\mathbf{t})$ is the Dirac $\delta$-function. Usually, the normalized transfer functions of high-speed photodiodes can be mathematically approximated by functions of two kinds, namely, by the exponential function $\exp (-\mathbf{t} / \mathbf{T})$ or the hyperbolic-like function $e^{e} \mathbf{1}+(\mathbf{t} / \mathbf{T})^{\mathbf{m}} \mathrm{ll}_{\mathrm{u}}^{-1}$ with the power $\mathbf{m} \in[\mathbf{1}, \mathbf{2}]$, where the characteristic parameter $\mathbf{T}$ is determined by properties of each individual type of photodetector. Figure 4 illustrates principally appearing the response function conditioned by the incoming Gaussian optical pulse and the exponential transfer function.

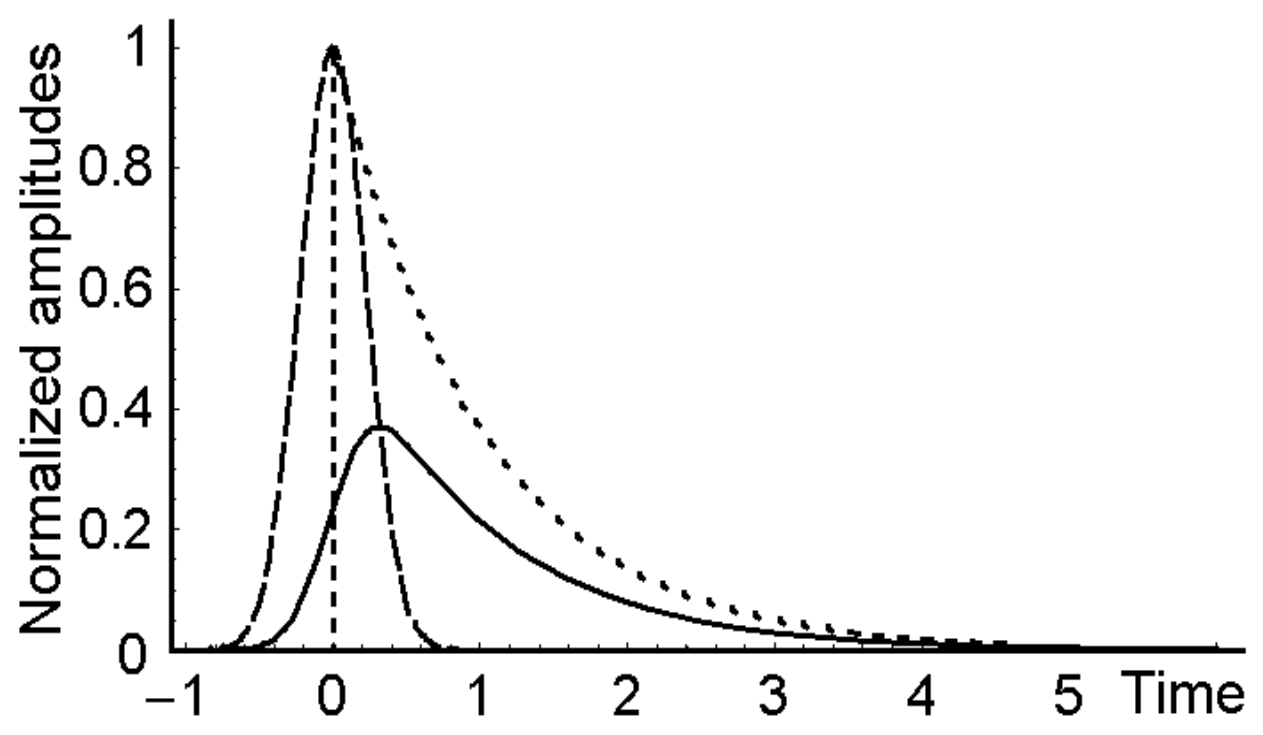

Figure 4. Shaping the response function (solid line) conditioned by the incoming ultra-short Gaussian optical pulse (dashed line) and the exponential transfer function (dotted line); the scales of curves are changed to illustrate better.

\section{EXPERIMENTAL RESULTS}

Within using this detection system some measurements of ultra-short pulse continuous trains generated by single-mode semiconductor laser structures at the wavelength of $1320 \mathrm{~nm}$ and $1550 \mathrm{~nm}$ were done. Our experimental studies have demonstrated that within mode-locking single-mode InGaAsP-laser heterostructures at a threshold of self-excitation (practically, it was realized at a pump current of about $50 \mathrm{~mA}$ ), only a spike-mode free oscillation regime had been observed with an individual spike width of about $0.7-0.9$ ps. The investigation of these spikes has shown that each 
individual spike includes an irregular set of intensity fluctuations. Figure 5 represents an example of the digitized oscilloscope trace for the auto-correlation function related to a spike-mode free oscillation when an average spike width is close to $0.7 \mathrm{ps}$.

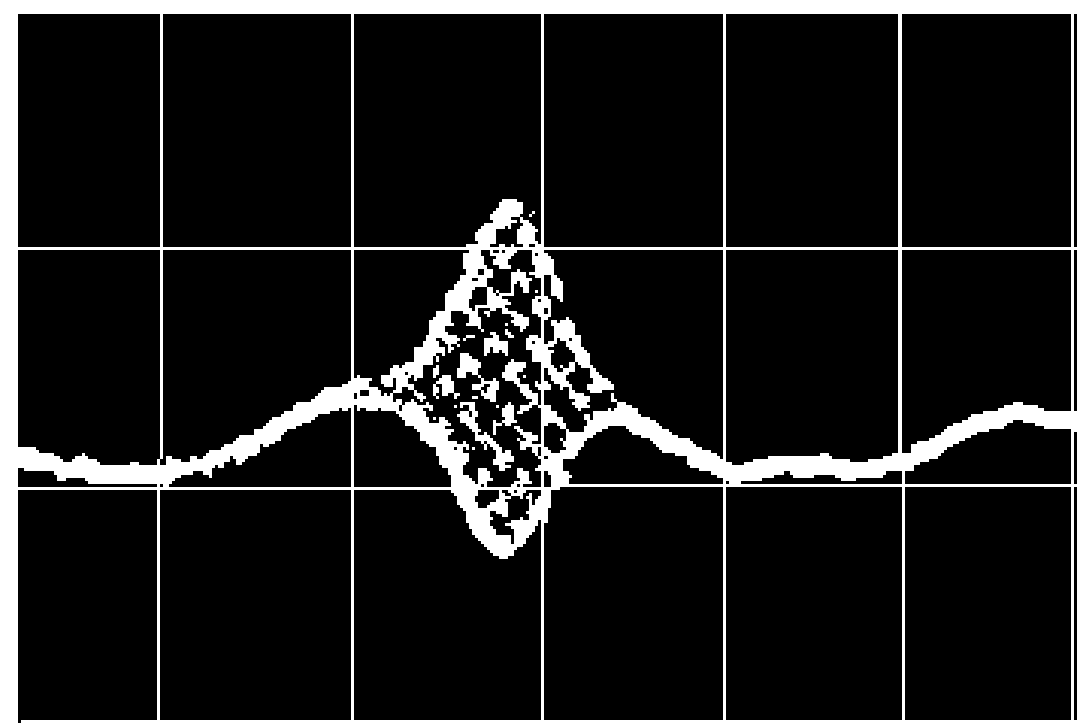

Figure 5. The digitized oscilloscope trace for the auto-correlation function for a spike-mode free oscillation with an average spike width of about $0.7 \mathrm{ps}$.

Shaping a continuous sequence of stable regular ultra-short optical pulses with duration of about $2-10$ ps can be achieved only after exceeding a threshold of self-excitation by $10-20 \%$. In so doing, one can observe increasing the energy of oscillation about 10 times, so that the peak-power of regular optical pulses approaches $0.2-1.0 \mathrm{~W}$.

Figure 6 demonstrates an example of the digitized oscilloscope trace for the auto-correlation function related to a regular pulse sequence when an average pulse width is about $6.3 \mathrm{ps}$.

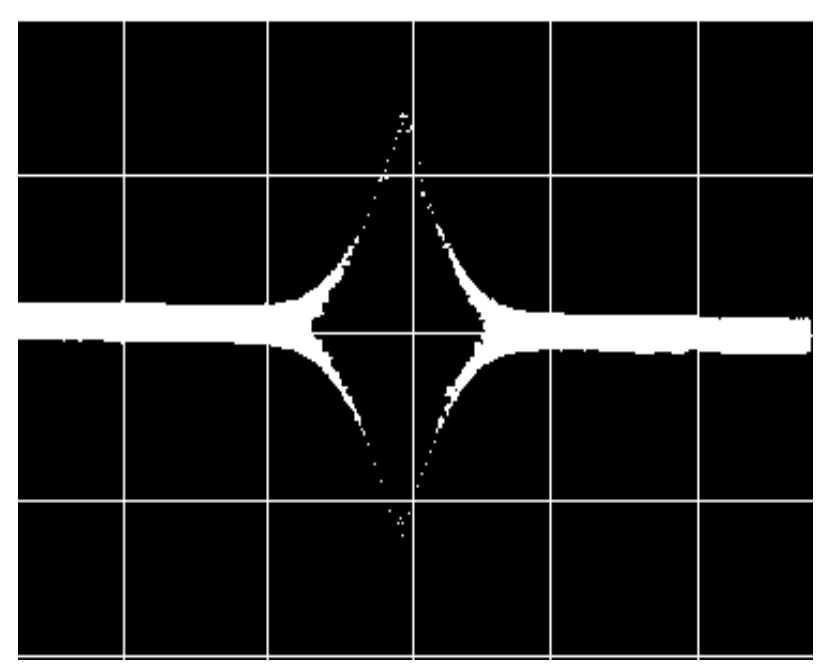

a.

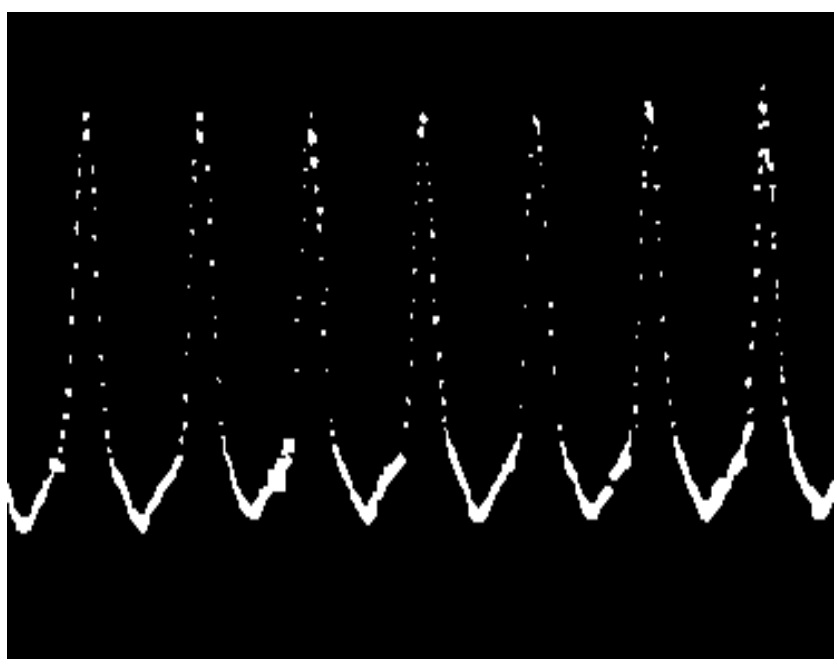

b.

Figure 6. The digitized oscilloscope traces related to a regular pulse train: (a) the auto-correlation function for an average pulse width of about $6.3 \mathrm{ps}$; (b) the output signal from a high-speed photodetector. 


\section{CONCLUSION}

Evidently, the proposed approach makes it possible to consider the initial stage of the active mode-locking in semiconductor laser structures through analyzing the properties of dispersion relations in terms of stability for small initial perturbations. Within such an analysis, the nonlinear process of shaping optical pulses can be interpreted as manifesting instability of diffusion type. We have observed both the stage of spike-mode oscillation and the stage of pulse shaping. Results of the performed analysis are in coincidence with both the data of numerical simulations and the obtained experimental data

\section{ACKNOWLEGMENTS}

This work has been financially supported by the CONACyT, Mexico: project \# 61237-F.

\section{REFERENCES}

1. O Svelto. Principles of Lasers. (Springer, New-York, 1998).

2. J.P. van der Ziel. The mode-locking in semiconductor lasers. // Semiconductors \& Semimetals, Vol. 22 ( Lightwave Communication Technology), Volume Editor W.T. Tsang (Academic Press, Orlando, 1985) Chapter 1.

3. H. A. Haus. Theory of mode-locking with a fast saturable absorber, J. Appl. Phys., Vol.46, no.7, pp 3049-3058, (1975).

4. H.A. Haus Mode locking of semiconductor laser diodes. Japan J. Appl. Phys., Vol.20, no.6, pp 1007-1020, (1981).

5. G. B Whitham.. Linear and Nonlinear Waves J.Wiley \& Sons, New-York, (1974).

6. R. K Dodd., J. C.Eilbeck, J. D.Gibbon and H. C Morris,. Solitons and Nonlinear Wave Equations. (Academic Press, London, 1982).

7. H.A.Haus. Waves and Fields in Opto-Electronics..( Prentice-Hall, Upper Saddle River, N.J., 1984). 